\title{
Standounkt
}

Beim Klimagipfel in Kopenhagen ist ein Durchbruch nötig

\section{Jetzt oder nie}

\author{
Der Erfolg des Klimagipfels von Kopenhagen steht auf der \\ Kippe. Viele Akteure scheinen an einem Deal um jeden Preis \\ interessiert als an einem rechtlich verbindlichen Abkommen, \\ das Klimageschichte schreibt. Die Welt wartet auf den Start- \\ schuss für eine technologische und gesellschaftliche Revolution \\ - die politischen Maßnahmen dafür müssen im Dezember \\ beschlossen werden. Von Christoph Bals
}

N ach dem Stillstand der letzten Monate ist endlich Bewegung in die Klimaverhandlungen der Vereinten Nationen gekommen. Seit der jüngsten Verhandlungsrunde in Bangkok und Barcelona liegen die Optionen in einer Form auf dem Tisch, dass nun ernsthaft über sie verhandelt werden kann. Doch große Fragen bleiben: Ist auf internationaler Ebene ausreichend politischer Wille vorhanden, um ein gerechtes und ambitioniertes Abkommen zu erreichen? Zu welchen Klimaschutzzielen und Finanzleistungen können sich die Industrieländer durchringen? Sind die Ölstaaten um Saudi-Arabien weiterhin in der Lage, den Verhandlungsprozess in Ihrem Sinne zu blockieren? Und zu guter Letzt muss die Frage gestellt werden, ob die USA international überhaupt handlungsfähig sein werden, bevor das von Präsident Obama eingebrachte nationale Klimaschutzgesetz den Senat passiert hat?

\section{Merkels Wandel}

Aber nicht nur im Verhandlungsprozess selbst, sondern auch auf nationaler Ebene sind viele Punkte unklar. In Deutschland geht es darum, ob Kanzlerin Merkel wieder eine Führungsrolle im internationalen Klimaschutz übernehmen wird, oder ob Deutschland, wie in den letzten Monaten, sich eher als farbloser Mitläufer etabliert. Im Jahr 2007 hatte sie diese Rolle als Präsidentin der Europäischen Union und der G8 beherzt übernommen. Dann aber wurde sie, nach heftigem Druck der Auto- und Energiekonzerne, wieder deutlich zögerlicher. Die Energieeffizienzgesetzgebung in Deutschland scheiterte, mit der nicht ökologisch ausgestalteten Abwrackprämie wurde die große Chance verpasst, einen Strukturwandel in der Autoindustrie zu befördern. Und die Kanzlerin schaute $\mathrm{zu}$, wie die Kohleindustrie munter neue Kohlekraftwerke plante und zu bauen begann.

Welche Rolle wird Deutschland bei den drei großen Fragen des Klimaschutzes übernehmen? Erstens bezüglich der Ambition des Klimaschutzes: Wird sich die neue Regierung zumindest zum 40prozentigen Reduktionsziel bis 2020 auf der Basis von 1990 bekennen? Wird sie bereit sein, noch etwas draufzulegen, wenn von den großen Industrie- und Schwellenländern gefordert werden wird, ihre Ambition noch zu erhöhen? Viel wichtiger aber: Wird sie bereit sein, sechs bis zehn Milliarden Euro jährlich für den Klimaschutz, den Regenwaldschutz sowie die Unterstützung der besonders betroffenen Staaten und Menschen auf den Tisch zu legen? Hier hat sich Deutschland bisher zu keiner klaren Position durchgerungen und möchte das Geld zudem von dem schon vor Jahren zugesagten Mitteln für die internationale Armutsbekämpfung abzweigen. Klar ist: ohne Geld kein Deal und ohne angemessenes Geld kein ambitioniertes Abkommen.

Keiner sollte sich über die Größe der vor uns liegenden Aufgabe hinwegtäuschen. Es geht hier nicht darum, irgendein Umweltproblem zu beheben. Son- dern es geht vielmehr darum, ob wir innerhalb weniger Jahrzehnte von den fossilen Energieträgern, den Grundstoffen, die den Motor des weltweit vorherrschenden Wohlstandsmodells antreiben, Abschied nehmen. Es geht um nicht weniger als eine technologische Revolution, die mit deutlich veränderten Verhaltensmustern einhergehen muss. Dass dieser Umstieg schwierig und mit zahlreichen Widerständen verbunden ist, sollte keinen wundern. Aber jetzt gilt es, die Wende hin zu einem treibhausgasfreien Wohlstandsmodell einzuleiten. Wenn dies nicht gelingt, stehen uns viel größere Risiken und Umbrüche bevor. Und dann blieben die Chancen für Arbeitsplätze, Innovation und weltweite Kooperation, die für uns in einem zügigen Umbau ins Solarzeitalter liegen, ungenutzt.

\section{Abschied von fossilen Energieträgern}

Bis zum Klimagipfel in Kopenhagen werden sich die Regierungschefs und Minister weltweit in unterschiedlichen politischen Foren mit dem Thema auseinander gesetzt haben. Sie müssen den Verhandlern erweiterte Mandate geben, damit Kompromisse zwischen Industrieund Entwicklungsländern erreicht werden. Es wäre fatal, wenn das Kompromisse mit dem Klima wären, denn dieses lässt nicht mit sich verhandeln. Kopenhagen steht für eine Weggabelung der Menschheit. Zum jetzigen Zeitpunkt würde es auch nicht mehr überraschen, wenn eine Verhandlungsverlängerung um einige Monate notwendig werden wird, damit ein gerechtes und ambitioniertes Abkommen möglich wird. Wenn der Klimagipfel in Kopenhagen nicht zur Sterbehilfe für die Eispanzer auf Grönland werden soll, muss die internationale Politik in den nächsten Monaten über sich hinauswachsen.

I AUTOR + KONTAKT

Christoph Bals ist Politischer Geschäftsführer von Germanwatch.

Germanwatch e.V., Dr.Werner-Schuster-Haus, Kaiserstr. 201, 53113 Bonn. Tel.: +49 228 60492-17, Fax +49 228/60492-19, E-Mail: bals@germanwatch.org 
(c) 20I0 Authors; licensee IÖW and oekom verlag. This is an article distributed under the terms of the Creative Commons Attribution Non-Commercial No Derivates License (http://creativecommons.org/licenses/by-nc-nd/3.o/), which permits unrestricted use, distribution, and reproduction in any medium, provided the original work is properly cited. 